\title{
Relations between Interstellar medium tracers in galaxies
}

\author{
G. Galletta ${ }^{1}$, V. Casasola ${ }^{1,3}$, L. Piovan ${ }^{1,4}$, E. Merlin $^{1}$, and D. Bettoni ${ }^{2}$ \\ 1 Dipartimento di Astronomia, Università di Padova, Vicolo dell'Osservatorio 2, 35122 Padova, Italy \\ e-mail: [giuseppe.galletta;viviana.casasola]@unipd.it; [piovan;merlin]@pd.astro.it \\ 2 INAF - Osservatorio Astronomico di Padova, Vicolo dell 'Osservatorio 5, 35122 Padova, Italy \\ e-mail: daniela.bettoni@oapd.inaf.it \\ 3 Observatoire de Paris-LERMA, 61 Av. de l'Observatoire, 75014 Paris, France \\ 4 Max-Planck-Institut für Astrophysik, Karl-Schwarzschild-Str. 1, Garching bei München, Germany \\ e-mail: piovan@mpa-garching.mpg.de
}

Received 20 April 2006 / Accepted 20 September 2006

ABSTRACT

\begin{abstract}
Aims. We study the relations existing between fluxes emitted at the $\mathrm{CO}(1-0)$ line, 60 and $100 \mu \mathrm{m}$ wavelengths, and the $\mathrm{B}$ and soft $\mathrm{X}$-ray wavebands for galaxies of all morphological types. The large set of data that we created allowed us to revisit some of the already known relations between the different tracers of the interstellar medium (ISM): the link between the FIR flux and the CO line emission, the relation between X-ray emission in non-active galaxies and the blue or FIR luminosity.

Methods. Using both catalogues of galaxies and works in the literature, we collected fluxes in the FIR, $21 \mathrm{~cm}$, and $\mathrm{CO}(1-0)$ lines and in the soft X-ray for two samples, consisting of normal and interacting galaxies, respectively. By joining these samples, we have data for a total of 2953 galaxies, not all observed in these four wavebands.

Results. All the relations found are discussed in the frame of the star formation activity that is the link for most of them. We note that when an active star formation is present, it may link the galaxy fluxes at almost all wavelengths, from $\mathrm{X}$ to microwaves. In contrast, in early-type galaxies where the current star formation rate has faded, the X-FIR fluxes link disappears. This result for early-type galaxies is discussed and explained in detail in the frame of a suitable theoretical model, obtained by coupling chemo-dynamical $N$-body simulations with a dusty spectrophotometric code of population synthesis.
\end{abstract}

Key words. galaxies: ISM - galaxies: fundamental parameters - infrared: ISM - radio lines: ISM - X-rays: ISM

\section{Introduction}

The observations of galaxies at various wavelengths, going from radio to X-ray, allow the study of the relationships between the various phases of the interstellar gas and between gas, dust, and stars. Some of these relations have already been known for many years, such as the one between CO and far-infrared (FIR) luminosities (Sanders \& Mirabel 1985; Sanders et al. 1986; Solomon \& Sage 1988; Devereux \& Young 1991). Others, connected with X-ray emission, have been studied more recently (Griffiths \& Padovani 1990; David et al. 1992; Ranalli et al. 2003).

At present, different tracers of the gas are known, such as millimetric lines for the cold molecular gas, the $21 \mathrm{~cm}$ line for atomic hydrogen at $\sim 100 \mathrm{~K}$, IR bands for molecules at thousands of degrees, UV lines, and X-ray emission for hotter gas. The dust distribution is also traced by FIR emission at 60 and $100 \mu \mathrm{m}$, if the grains are warm (Bregman et al. 1992), or at $170 \mu \mathrm{m}$, if they are colder (Popescu et al. 2002). The diffusion of large archives of observations at the above wavelengths (except for molecular lines) has allowed the compilation of catalogues in the past years containing a huge number of galaxies. Using these catalogues and the works presented in the literature, we collected fluxes in FIR, $21 \mathrm{~cm}, \mathrm{CO}(0-1)$ line, and soft X-ray for two broad samples of normal (Bettoni et al. 2003a) and interacting (Casasola et al. 2004a) galaxies. By joining these samples, we have amassed data for a total of 2953 galaxies, not all observed in these four wavebands. The fluxes measured with the different tracers now allow a study of the link between dust, gas, and stars based on hundreds of galaxies.

It is known that the fluxes emitted by a galaxy at very different wavelengths may be linked by means of the star formation mechanism (see David et al. 1992; Ranalli et al. 2003). For instance, the formation of massive stars generates heating of the dust clouds they are embedded in, by absorption of their UV radiation, and produces a re-emission of this energy in the FIR. This process links the current star formation rate to the IR emission at 60 and $100 \mu \mathrm{m}$ (Thronson \& Telesco 1986). The ionizing radiation of stars may also produce evaporation of the molecular clouds. Inside these clouds, where the particle density is high enough to produce a significant number of collisions between $\mathrm{H}_{2}$ and $\mathrm{CO}$ molecules, these are excited and produce photons, but in optically thick regions. The warming by the UV stellar light makes these regions less dense, thereby making visible the CO lines at their edge. Because of this mechanism, these lines are considered tracers of the cold molecular hydrogen that does not emit observable lines. The newly formed stars are also responsible for the $\mathrm{X}$-ray emission produced by very massive stars, by core-collapse SN, and by high-mass X-ray binaries. According to the mechanisms described above, we expect that galaxies with active star formation will have a FIR emission, but also $\mathrm{CO}$ and X-ray emissions induced by the more massive stars, linked by different kinds of relations.

When the star formation decreases or vanishes, the FIR emission decreases as well, but it may be fed by the stellar light absorbed and re-emitted in the infrared by dust (cirrus), while 

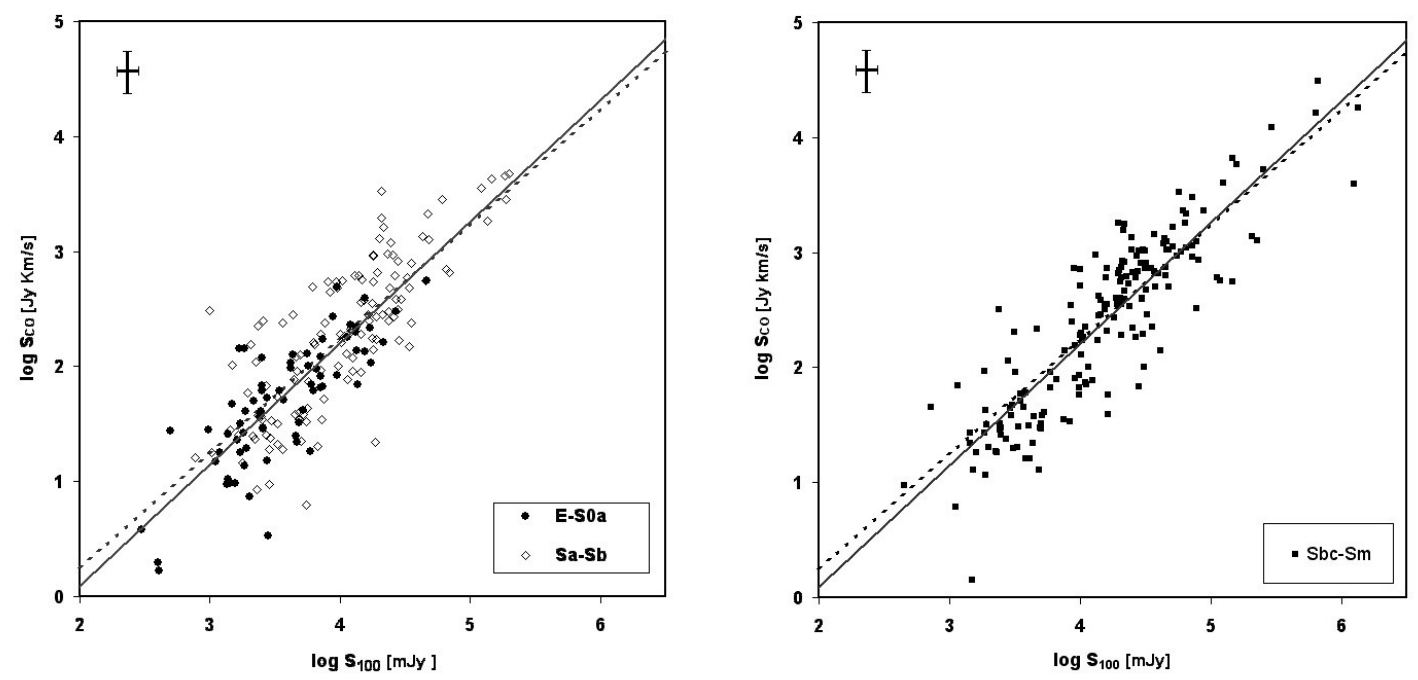

Fig. 1. The relation between the flux from the $\mathrm{CO}(1-0)$ line and at $100 \mu \mathrm{m}$, plotted as a full line, with the representative points of the single galaxies plotted with different symbols. The crosses at the upper left of the figures represent the mean errors for the fluxes in the whole sample. For comparison, the relation found by Bregman et al. (1992) for a sample of early-type galaxies is shown as a dotted line. The early-type galaxies and early spirals from $\mathrm{E}$ to $\mathrm{Sb}$ are plotted in the left panel, while the late spirals are plotted in the right panel.

low-mass X-ray binaries and type I SN contribute to the high energy galaxy spectrum. In addition, AGB stars, surrounded by dust, and the cooling flows of the interstellar medium ejected by supernovae may together produce additional IR and X emission.

To study the activity of the galaxies at different wavebands, we collected data on galaxies starting from the original data on fluxes in the $60,100 \mu \mathrm{m}, \mathrm{CO}(1-0)$ lines, and in the soft X-ray used to compile our catalogues (Bettoni et al. 2003b; Casasola et al. 2004b). The merging of these two catalogues produces 1764 known values of FIR fluxes (1837 have $100 \mu$ m flux), 391 soft X-ray fluxes, and 434 values for the $\mathrm{CO}(1-0)$ line luminosity. We extracted the values of the distance moduli, blue absolute magnitudes, and morphological classification for all of them from the LEDA catalogue (Paturel et al. 1997).

These are 1038 galaxies with evident signs of interactions or disturbed morphologies according to the catalogues of Arp (1966), Arp \& Madore (1987), and Vorontsov-Velyaminov (1959). We shall refer to them as "perturbed galaxies". The remaining 1915 galaxies that appear neither morphologically nor dynamically perturbed are called "normal galaxies". In our sample, we have 253 galaxies that have a spectral classification for the nucleus, and 231 of these appear to host an AGN (Seyfert 1,2 or transition type, Seyfert 3 or Liners) according to the classifications of Ho et al. (1997) and Véron-Cetty \& Véron (2003). Most of the remaining 2722 galaxies lack information about nuclear spectrum or have spectra of HII regions (22 starburst spectra). They are not included in any AGN catalogue and, for this reason, we refer to them in the following discussion as "non active galaxies" and to the others as "active galaxies". With all these data, we crossed the various tracers to understand and revisit the main relations existing between $\mathrm{X}, \mathrm{FIR}, \mathrm{CO}$, and B luminosities.

\section{Cold gas and warm dust}

The relations existing between different cold components of the ISM, such as the molecular gas and the dust, have been studied for many years (Sanders \& Mirabel 1985; Solomon \& Sage 1988; Bregman et al. 1992). They find that the global galaxy luminosity derived from $\mathrm{CO}(1-0)$ line is directly related with the flux at $100 \mu \mathrm{m}$. With our large sample we tested these relations using galaxies of different morphological types and activity or interaction.

In Fig. 1 we plotted the logarithm of the flux measured from $\mathrm{CO}(1-0)$ line vs. the logarithm of the IRAS flux at $100 \mu \mathrm{m}$. In our plots, we have 193 galaxies with classification from $\mathrm{E}$ to $\mathrm{Sb}$ and 178 from Sbc to Sm. The relation found by Bregman et al. (1992) for a sample of early-type galaxies, $\log S_{\mathrm{CO}}=\log S_{100}-$ 1.76 , is also plotted as comparison.

The relations are evident with this wider sample of galaxies. In these diagrams, active and non-active galaxies appear mixed together without clear differences and have been plotted together. The same behaviour appears for interacting and non interacting galaxies, which are not distinguished in our plots.

For all the galaxy types, we find:

$\log S_{\mathrm{CO}}=1.06 \log S_{100}+2.02$

with a correlation coefficient of 0.74 and an r.m.s. of 0.37 . In the above formula, $S_{100}$ is in mJy and $S_{\mathrm{CO}}$ is in $\mathrm{Jy} \mathrm{km} \mathrm{s}{ }^{-1}$. Similar relations exist between the CO fluxes and the FIR magnitudes, defined as:

$m_{\mathrm{FIR}}=-2.5 \log \left(2.58 S_{60}+S_{100}\right)+22.25$

where $S_{60}$ and $S_{100}$ the fluxes at 60 and $100 \mu \mathrm{m}$ respectively, are in $\mathrm{mJy}$. We find for all the galaxy types

$\log S_{\mathrm{CO}}=0.41 m_{\mathrm{FIR}}+6.86$

with a correlation coefficient of 0.69 and rms of 0.40 . The results are based on 179 early types and 170 late-type galaxies. These relations are not plotted in this paper due to their similarity with Fig. 1.

We note that irregular galaxies are not fitted by these relations but are widespread. In our sample there are just 10 galaxies and their representative points have not been plotted in Fig. 1.

\section{X-ray component}

We are interested in understanding what relations exist between $L_{\mathrm{X}}$, the X-ray luminosity, and the other global galaxy 

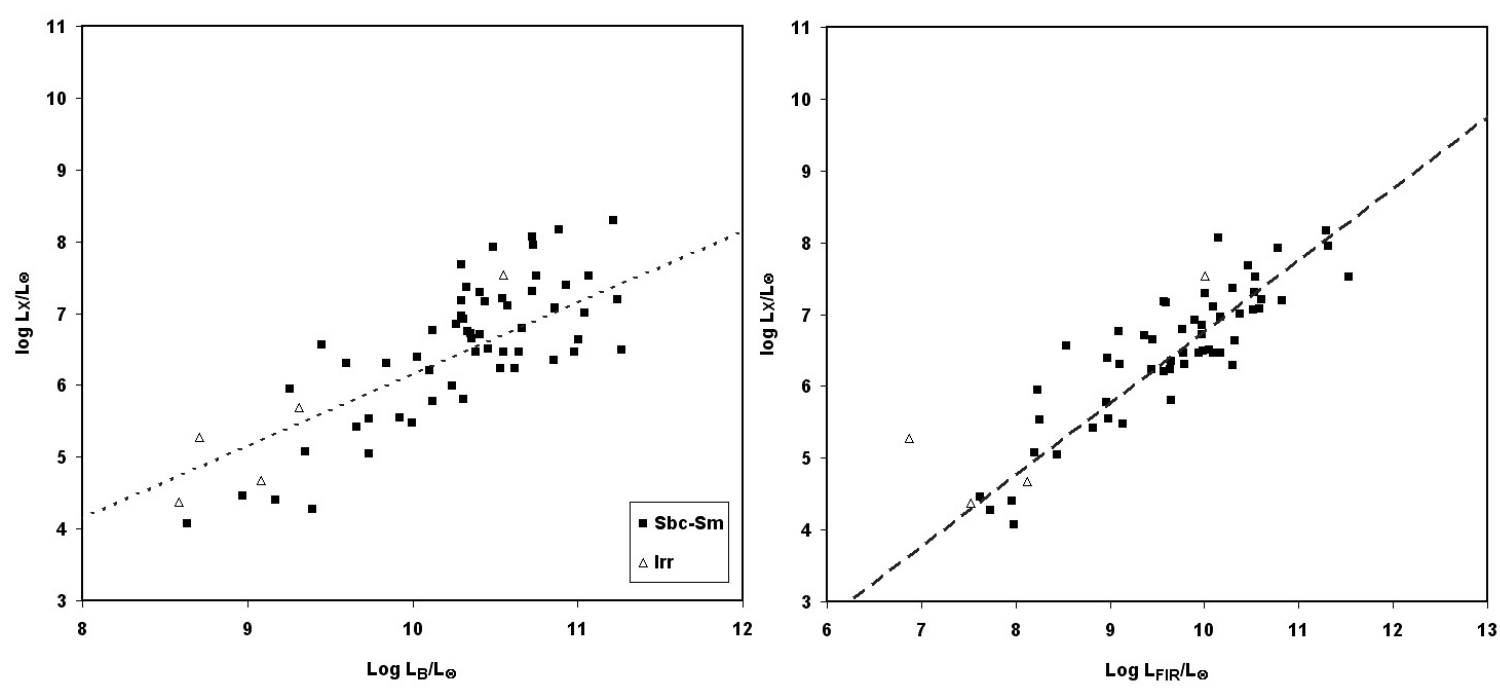

Fig. 2. Left: the X-ray luminosity plotted vs. blue luminosity, in solar units, for late-type, non-active galaxies. The relation corresponding to emission caused by discrete sources, indicated in Eq. (4) is plotted as a dotted line. Right: X-ray luminosity vs. FIR luminosity, in solar units for the late-type, non-active galaxies. The linear relation indicated in Eq. (7) is plotted as a dashed line.

properties. From the literature, it is known that a proportionality exists between $L_{\mathrm{X}}$ produced by discrete sources and $L_{B}$, the blue luminosity of the whole galaxy. This relation has been studied by Ciotti et al. (1991) and compared by Beuing et al. (1999) with soft X-ray fluxes measured by the ROSAT satellite. It appears that late-type galaxies have a global X-ray luminosity directly proportional to $L_{B}$, while early-type systems are dominated by emission produced by hot diffuse gas, and their $L_{\mathrm{X}}$ is proportional to the square power of the blue luminosity, as discussed by Beuing et al. (1999). For this reason, the early and late-type galaxies are discussed separately.

\subsection{Late-type galaxies}

With our data, the X-ray luminosity of galaxies with a morphological type later than $\mathrm{Sb}$ can be fitted by a linear relation as a function of $L_{B}$ (in Fig. 2, left panel). The direct proportionality is expressed by the equation:

$\log L_{X}=\log L_{B}-3.85$

with an rms from observed data of $\sigma=0.61$ based on 63 galaxies. In this formula and in the following, all the luminosities are expressed in solar units.

If, instead of the blue luminosity, we use the galaxy area $D_{\mathrm{kpc}}^{2}$, calculated from the apparent diameter measured at the $25 \mathrm{mag} \operatorname{arcsec}^{2}$ isophote and converted in $\mathrm{kpc}^{2}$, we discover that the relation is still present, but with a wider spread. It becomes

$\log L_{\mathrm{X}}=\log D_{\mathrm{kpc}}^{2}+3.83$

( $\sigma=0.80$ ) for a sample of 64 galaxies.

A relation similar to that of Ciotti et al. (1991) has been found by some authors (Griffiths \& Padovani 1990; David et al. 1992; Ranalli et al. 2003), but using $60 \mu \mathrm{m}$ fluxes or FIR luminosities. The values of $L_{\mathrm{FIR}}$ are calculated using the formula

$\log L_{\mathrm{FIR}}=2.59+\log \left(2.58 S_{60}+S_{100}\right)+2 \log d$

where $L_{\mathrm{FIR}}$ is in solar luminosities, fluxes are in $\mathrm{mJy}$, and the galaxy distance $d$ is in Mpc.
From our data it is possible to find a relation between $L_{X}$ and $L_{\mathrm{FIR}}$ that fits the values of late-type galaxies. We found $L_{\mathrm{X}} \propto$ $L_{\mathrm{FIR}}^{0.90}$, similar to the $L_{\mathrm{X}} \propto L_{\mathrm{FIR}}^{0.88}$ found by Ranalli et al. (2003) for fluxes between 0.5 and $2 \mathrm{keV}$ and to the $L_{\mathrm{X}} \propto L_{\mathrm{FIR}}^{0.95}$ found by David et al. (1992) using fluxes between 0.5 and $4.5 \mathrm{keV}$. Forcing the relation to a linear proportionality between $L_{\mathrm{X}}$ and $L_{\text {FIR }}$ we find:

$\log L_{\mathrm{X}}=\log L_{\mathrm{FIR}}-3.18$

with a $\sigma$ of 0.47 , based on 147 galaxies. This relation is plotted in the right panels of Figs. 2 to 4 .

We note that the $B$ and FIR luminosities are also connected in late-type galaxies by means of a linear relation fitted by:

$\log L_{\mathrm{FIR}}=\log L_{B}-0.38$

with $\mathrm{rms}=0.5$. This equation, inserted into the relation (4) gives

$\log L_{\mathrm{X}}=\log L_{\mathrm{FIR}}-3.47$,

similar to the result of Eq. (7) and to that found by Ranalli et al. (2003). This is an independent way to confirm our results and to verify the existence of a global link between $L_{\mathrm{FIR}}, B$ light, and $\mathrm{X}$-ray emission. The connection between $B$ luminosity or galaxy area and $\mathrm{X}$ or FIR luminosities will be discussed in Sect. 5 .

\subsection{Early-type galaxies}

When the early-type galaxies are considered in the relations involving X-ray emission, the correlations become less evident. Considering soft $\mathrm{X}$-ray and $B$ luminosities, we find a relation

$\log L_{X}=2 \log L_{B}-13.57$

( $\sigma=0.73$ ) based on 224 galaxies and plotted in the left panel of Fig. 3. The above formula agrees with the expected relation for X-ray emission coming from hot diffuse gas, as discussed by Beuing et al. (1999).

The relation still holds if $D_{\mathrm{kpc}}^{2}\left(\mathrm{kpc}^{2}\right)$ is used. It becomes

$\log L_{\mathrm{X}}=2 \log D_{\mathrm{kpc}}^{2}+1.51$

$(\sigma=0.85)$ for 226 early-type galaxies from $\mathrm{E}$ to $\mathrm{Sb}$. 

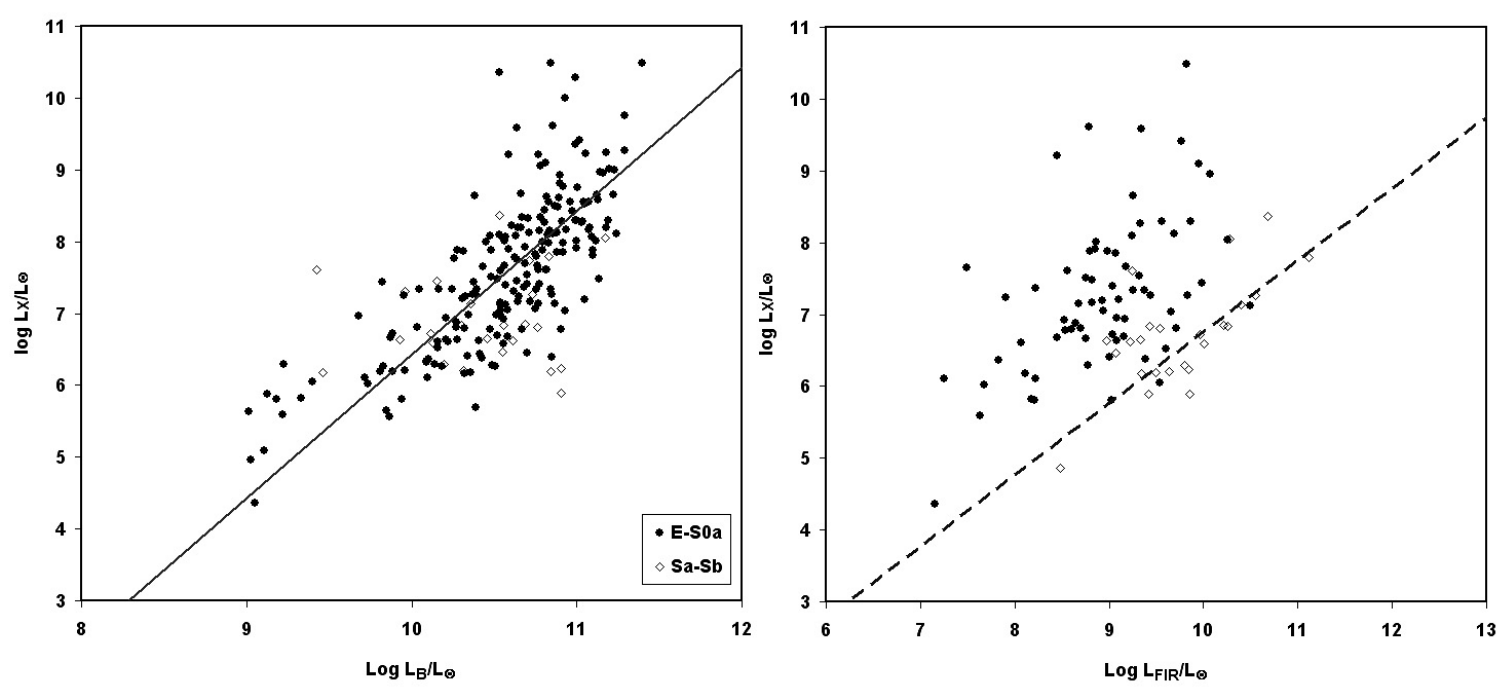

Fig. 3. Left: the X-ray luminosity plotted vs. blue luminosity, for early-type, non-active galaxies. The relation corresponding to emission caused by diffuse gas, valid for early-type galaxies and indicated in Eq. (10) is plotted as a full line. Right: X-ray luminosity vs. FIR luminosity for the early-type galaxies. The linear relation for late-type, non-active galaxies indicated in Eq. (7) is plotted as a dashed line.
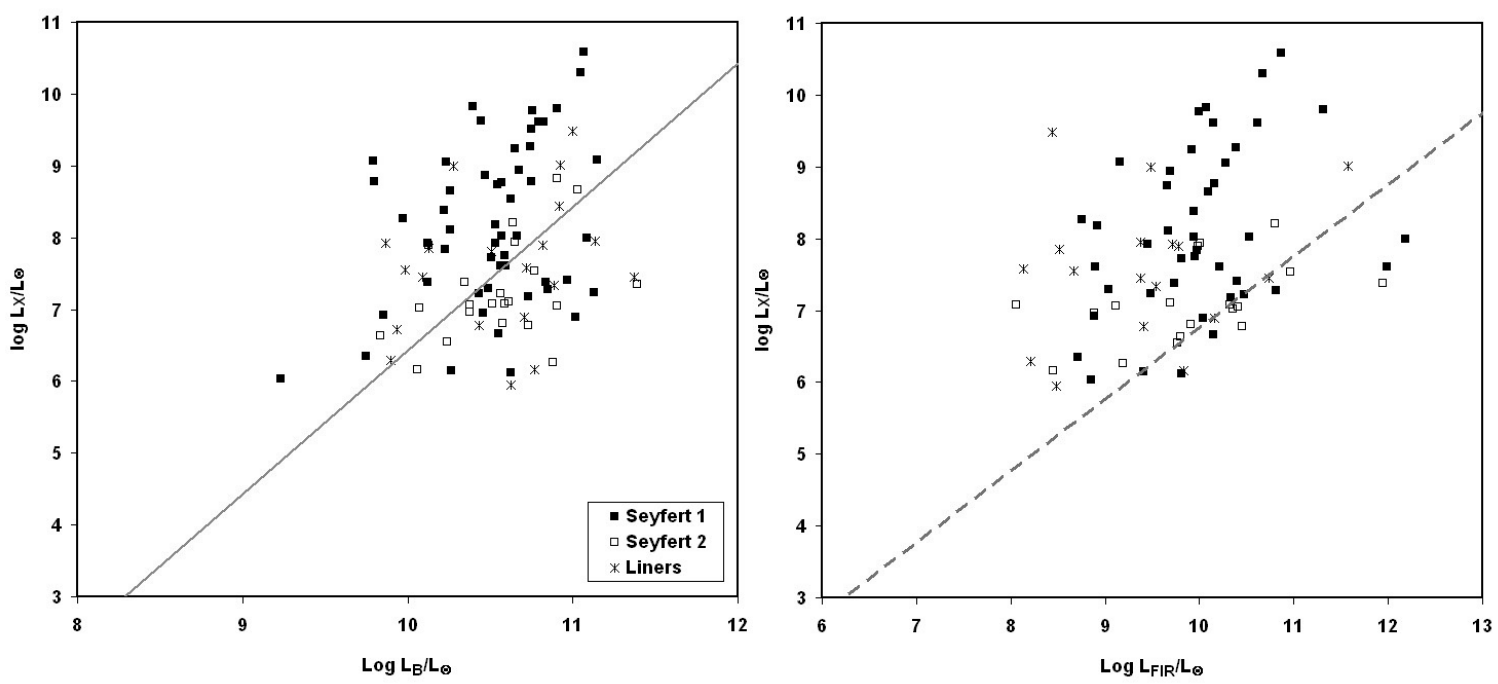

Fig. 4. Left: the X-ray luminosity plotted vs. blue luminosity for active galaxies, separated according to the type of activity. The relation corresponding to emission caused by diffuse gas, valid for early-type galaxies and indicated in Eq. (10) is plotted as a full line. Right: X-ray luminosity vs. FIR luminosity for the active galaxies. The linear relation for late-type galaxies indicated in Eq. (7) is plotted as a dashed line.

Many galaxies with high blue luminosity, indication of high masses and of a recent star formation, lie quite far from the mean line, with different behaviour than late-type galaxies.

If the X-ray fluxes are compared with FIR luminosity, the disagreement with the behaviour found in late-type galaxies is more evident. The plot $L_{\mathrm{X}}$ vs. $L_{\mathrm{FIR}}$ for early-type galaxies shows the representative points of the galaxies above the relation (7) for late-type galaxies (Fig. 3, right panel). To understand this apparent disagreement, we should use a theoretical analysis of the FIR emission, as explained in Sect. 4.

\subsection{Active galaxies}

Active galaxies (Seyfert 1, Seyfert 2, and Liners) have X-ray, $\mathrm{B}$, and FIR fluxes that are not linked. This happens because, to the emission mechanisms stimulating the light emission at the different wavebands described for non active galaxies, an X-ray emission comes from nucleus. In fact, the points representative of these active galaxies are spread in the plot over the discrete source line and around the diffuse gas line (see Fig. 4, left side). In the $L_{\mathrm{X}}-L_{\mathrm{FIR}}$ diagram (Fig. 4, right side) the spread is similar to that of early-type galaxies plotted in Fig. 3, but we separately plotted the active galaxies because of the particular nature of their X-ray emission, due to the nuclear contribution.

\section{Modelling $L_{X}, L_{B}$, and $L_{F I R}$ of early-type galaxies}

To cast light on the nature of the relations observed between $L_{X}$, $L_{B}$, and $L_{\mathrm{FIR}}$ for early-type galaxies, one has to consider the various components of a galaxy (stars, gas, and dust) and to understand their mutual interactions as far as the spectral energy distribution (SED) is concerned. There are two basic schemes for modelling the formation and evolution of early type galaxies: (1) the semi-analytical models on which a great deal of our 
understanding of the chemo-spectro-photometric properties is derived; and (2) the $N$-Body Tree-SPH simulations that, in contrast, have been used only occasionally to study spectrophotometric properties of early type galaxies. In the following part of this section we proceed as follows. First, we analyse the drawbacks of semi-analytical models, in particular dealing with the calculation of the infrared emission of early-type galaxies. Second, we discuss how dynamical simulations and a dusty spectrophotometric code, when mixed together, allow us to move forward a step in the calculations of the SEDs properties. Third, we show in detail how our model has been built and the coupling between dynamics and dusty population synthesis was done.

\subsection{The semi-analytical models and their drawbacks}

The semi-analytical models approximate a galaxy to a pointmass system in which gas is turned into stars by means of suitable recipes for star formation, and heavy elements are produced by stellar nucleosynthesis and stellar winds/explosions. The standard evolutionary population synthesis technique (EPS) is usually applied to derive the SED of the galaxy, with models able to explain many global features of early type galaxies, as amply described by many authors (Arimoto \& Yoshii 1987, 1989; Bressan et al. 1994; Gibson 1997; Tantalo et al. 1996, 1998). There are three important and problematic issues of these models to be discussed for our purposes.

First, to determine the age at which the galactic wind sets (Larson 1974; Larson \& Dinersten 1975), we need some hypothesis about dark and baryonic matter with their relative distributions and about the heating and cooling efficiency of the various mechanisms to properly evaluate the total gravitational potential well and to describe the thermal history of the gas. In this scheme it turns out that the galactic wind occurs typically for ages $t_{\mathrm{GW}}<1 \mathrm{Gyr}$, later in a massive early-type galaxy and much earlier in galaxies of lower mass (Arimoto \& Yoshii 1987, 1989; Bressan et al. 1994; Gibson 1997; Tantalo et al. 1996, 1998; Chiosi et al. 1998). The maximum duration of star-forming activity therefore follows the trend $\Delta t_{\mathrm{SF}} \propto M_{\mathrm{G}}$ in these models. This trend of the SFH is, however, contrary to what is required by the observed trend forward the $\alpha$-enhancement for early type galaxies, which implies that the maximum duration of the star forming activity should decrease when the galaxy mass increases $\left(\Delta t_{\mathrm{SF}} \propto M_{\mathrm{G}}^{-1}\right)$ (see Bressan et al. 1996; Kuntschner 2000; Trager et al. 2000a,b; Tantalo \& Chiosi 2004; Thomas et al. 2005, for more details on the enhancement in $\alpha$-elements and the SFH of early-type galaxies).

Second, after the galactic wind phase, star formation no longer occurs and the evolution is merely passive. However, AGB and RGB stars continue to lose gas in amounts that are comparable to those before the galactic wind (Chiosi 2000). What is the fate of this gas? One may imagine that the large amount of gas lost by stars will expand into the dark matter halo and heat up to an energy and overwhelm the gravitational potential, and will escape the galaxy. Most likely a sort of dynamical equilibrium is reached in which gas is continuously ejected by stars and lost by the galaxy. It may happen therefore that some amount of gas is always present in the galaxy. The question is not obvious because, if an early type galaxy is free of gas and contains only stars, the SED is expected to drop off long ward of about $2 \mu \mathrm{m}$, and no IR emission should be detected. However, as already pointed out long ago by Guhathakurta et al. (1986) and Knapp et al. (1989) (see also Fig. 3), many early-type galaxies of the local universe emit in the IR. The origin of this flux in the
MIR/FIR is most likely due to the dust present in a diffuse ISM that, once emits at those wavelengths heated up by the galactic radiation field. Therefore to match the IR emission, one has to allow for some amount of diffuse ISM. An interesting question is therefore how much gas can be present today in an elliptical galaxy and how it is distributed across the galaxy. Even if we can correctly estimate the amount of gas ejected by stars, the fate of this gas goes beyond the possibilities of classical semi-analytical models.

As a third point, note that, when we fold many SSPs to calculate a galaxy SED using the classical EPS technique, we simply convolve their fluxes with the SFH of the galaxy. Many classical spectrophotometric semi-analytical models of galaxies are built in this way: there is no dust at the level of SSPs and again no dust at the level of the galaxy model (see e.g. Arimoto \& Yoshii 1987; Arimoto \& Tarrab 1990; Bruzual \& Charlot 1993; Tantalo et al. 1996; Kodama \& Arimoto 1997; Tantalo et al. 1998; Buzzoni 2002, 2005). To calculate the emission by dust, a higher level of sophistication is required for the model. Indeed, one has to develop a model in which the sources of radiation and the emitting/absorbing medium are distributed, so as to face and solve the problem of the radiative transfer simulating the interactions in a realistic way among the various physical components of a galaxy. Among recent models of this kind are those by Silva et al. (1998), Devriendt et al. (1999), and Takagi et al. (2003).

\subsection{Improving upon semi-analytical models}

Two drawbacks of the semi-analytical models therefore concern (1) the description of galactic wind, which is supposed to occur within a finite time interval; and (2) the star formation history that is reversed, allowing longer SFH for more massive galaxies. These two problems, combined with a lack of geometrical information about the distribution of gas and dust, make semi-analytical models unsuitable properly for calculating the IR emission of early type galaxies. To improve upon them we need to use the results from dynamical simulations. They have been shown to be able to properly model the ejection of gas by the galaxy as a sort of continuous process, taking place whenever a gas particle heated up by various mechanism has acquired a higher velocity than the escape velocity (see e.g. Carraro et al. 1998; Kawata 2001; Springel 2001; Chiosi \& Carraro 2002). They are able to reproduce the SF history of early-type galaxies both in the context of the monolithic collapse scenario (Kawata 2001; Chiosi \& Carraro 2002) and recently in the context of hierarchical scenario (De Lucia et al. 2006). Finally, the galaxy is no longer a mass point, but a fully three-dimensional structure of the galaxy is available with spatial distribution of stars and gas.

With the aid of $N$-Body Tree-SPH simulations based on quasi-cosmological initial conditions in the standard-cold dark matter scenario (S-CDM), Merlin \& Chiosi (2006) modelled the formation and evolution of two early-type galaxies of different total mass (dark + baryonic matter in the cosmological proportions 9:1). The total masses under considerations are $1.62 \times 10^{12} M_{\odot}\left(\right.$ Model A) and $0.03 \times 10^{12} M_{\odot}($ Model B $)$. The galaxies have been followed from their separation from the global expansion of the universe to their collapse to virialized structures, the formation of stars, and subsequent nearly passive evolution. They are followed for a long period of time, i.e. 13 Gyr (Model A) and 5 Gyr (Model B), or at least well beyond the stages of active star formation that occurs within the first 3 to $4 \mathrm{Gyr}$ (see below). The models take into account radiative cooling by several processes, heating by energy feed back from supernova explosions (both types I and II), and chemical 
Table 1. Initial parameters for the dynamical simulations of Merlin \& Chiosi (2006) in the Standard CMD scenario, where masses are in units of $10^{12} M_{\odot}$, radii are in kpc, and ages are in Gyr.

\begin{tabular}{lll}
\hline \hline Model & $\mathrm{A}$ & $\mathrm{B}$ \\
cosmological background & $\mathrm{S}-\mathrm{CDM}$ & $\mathrm{S}-\mathrm{CDM}$ \\
\hline Initial redshift & 50 & 53 \\
$\Omega_{\mathrm{m}}$ & 1 & 1 \\
$H_{0}=50 \mathrm{~km} \mathrm{Mpc}^{-1} \mathrm{~s}^{-1}$ & 50 & 50 \\
Gas particles & 13719 & 13904 \\
$\mathrm{CDM}$ particles & 13685 & 13776 \\
Total Mass & 1.62 & 0.03 \\
Initial baryonic mass fraction & 0.10 & 0.10 \\
Present gas mass & 0.062 & 0.0004 \\
Present star mass & 0.091 & 0.0029 \\
$M_{\text {star }} / M_{\text {baryons }}$ & 0.556 & 0.82 \\
Initial radius & 33 & 9 \\
Half-Mass radius of stars & 7 & 1 \\
Half-Mass radius of DM & 52 & 15 \\
Effective radius of stars & 5.2 & 0.8 \\
Present virial radius & 300 & 41 \\
Axial ratio $b / a$ (stars) & 1.08 & 1.04 \\
Axial ratio $c / a$ (stars) & 1.07 & 1.00 \\
Axial ratio $b / a$ (Dark Matter) & 1.14 & 1.14 \\
Axial ratio $c / a$ (Dark Matter) & 1.17 & 0.96 \\
Age of the last computed model & 13 & 5 \\
\hline
\end{tabular}

enrichment. All the models conform to the so-called revised monolithic scheme, because mergers of substructures have occurred very early in the life of the galaxy. Some parameters and results of the two models are summarised in Table 1. Note that the shape of the resulting galaxies is nearly spherical both in dark matter and stars.

The third drawback of the classical semi-analytical model was the lack of a description of the dusty component, which for our purposes needs to be included. The semi-analytical chemospectro-photometric model developed by Piovan et al. (2006b) allows us to overcome this issue. It takes into account not only the geometrical structure of galaxies of different morphological types, but also the effect of dust in converting the UV and optical light in FIR radiation. In brief, the Piovan et al. (2006b) model follows the infall scheme, allows for the onset of galactic winds, and contains three main components: (i) the diffuse interstellar medium composed of gas and dust whose emission and extinction properties have been studied in detail by Piovan et al. (2006a); (ii) the large complexes of molecular clouds in which new stars are formed; and (iii) the stars of any age and chemical composition. The total gas and star mass provided by the chemical model are distributed over the whole volume by means of suitable density profiles, one for each component and depending on the galaxy type (spheroidal, disk, and disk plus bulge). The galaxy is then split into suitable volume elements to each of which the appropriate amounts of stars, molecular clouds and interstellar medium are assigned. Each elemental volume absorbs radiation from all other volumes and from the interstellar medium in between. The elemental volume also emits the absorbed light again and produces radiation by the stars that it contains. On the other hand, the star formation, the initial mass function, and the chemical enrichment of the Piovan et al. (2006b) model are very similar to those by Bressan et al. (1994), Tantalo et al. (1996), Tantalo et al. (1998), and Portinari et al. (1998).
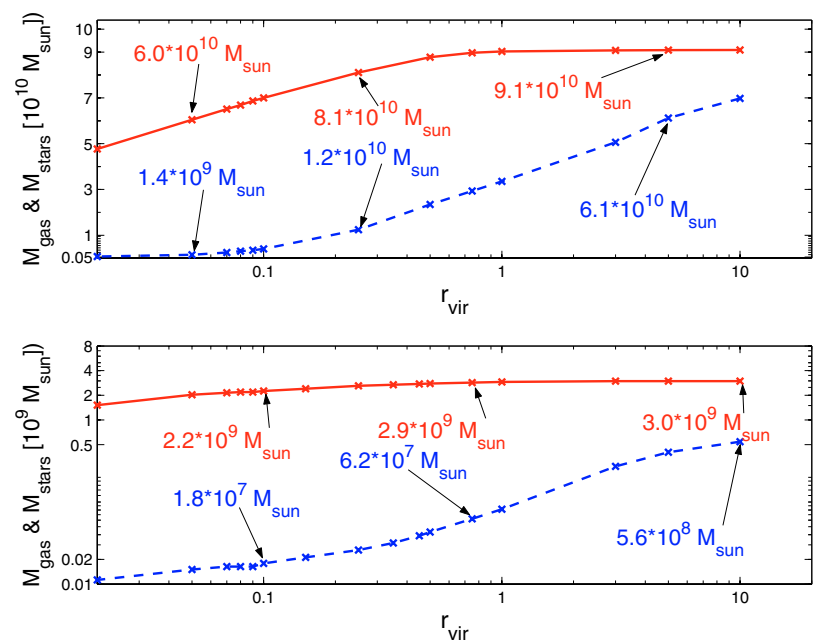

Fig. 5. Top panel: the masses of stars (continuous line) and gas (dashed line) for the dynamical model of $1.6 \times 10^{11} M_{\odot}$ baryonic mass as a function of the number of virial radii. The virial radius for this model is about $300 \mathrm{kpc}$. Bottom panel: the same as the upper panel, but for the dynamical model of baryonic mass $3.5 \times 10^{9} M_{\odot}$. For this model the virial radius is about $42 \mathrm{kpc}$.

\subsection{Coupling dynamical simulations and dusty population synthesis models}

The description of an early-type galaxy as far as predicting its spectro-photometric infrared properties can therefore be realised with a suitable combination of dynamical and spectrophotometric approaches. Coupling the dynamical models with spectro-photometric synthesis requires a number of steps that deserve some remarks.

\subsubsection{Radial density profiles}

Figure 5 shows the cumulative distribution of gas and stars as a function of the radial galactocentric distance normalized to the virial radius for model A (top panel) and model B (bottom panel). The gas is generally distributed in the external regions of the galaxy and steeply decreases inward. In contrast, the stars are more concentrated toward the centre. The gradients in the spherically averaged star- and gas- content provided by the dynamical models are the primary information to load into the spectro-photometric code of Piovan et al. (2006b). They allow us to infer the amount of gas contained within a given radius or within a given aperture. We fix the total dimension of the portion of the average model that produces the IR flux at a diameter $D_{\text {gal }}=25 \mathrm{kpc}$, consistent with the mean galaxy size of the observed sample.

As the spectro-photometric code of Piovan et al. (2006b) suited to describing early-type galaxies is written in spherical symmetry, we have to derive suitable spherical distributions for the density of stars and gas to be used into the model. The task is facilitated by the nearly spherical shape of the dynamical models. To this aim, we consider the sphere of radius $R_{\text {gal }}$ centred at the centre of mass of the stellar component. The sphere is then divided into a number of thin spherical shells whose derived average density of stars and gas is shown in Fig. 6. Even if the centre of mass of the star and gas distributions may not be exactly coincident, this is not relevant here, so the same coordinate centre can be used for both components. 


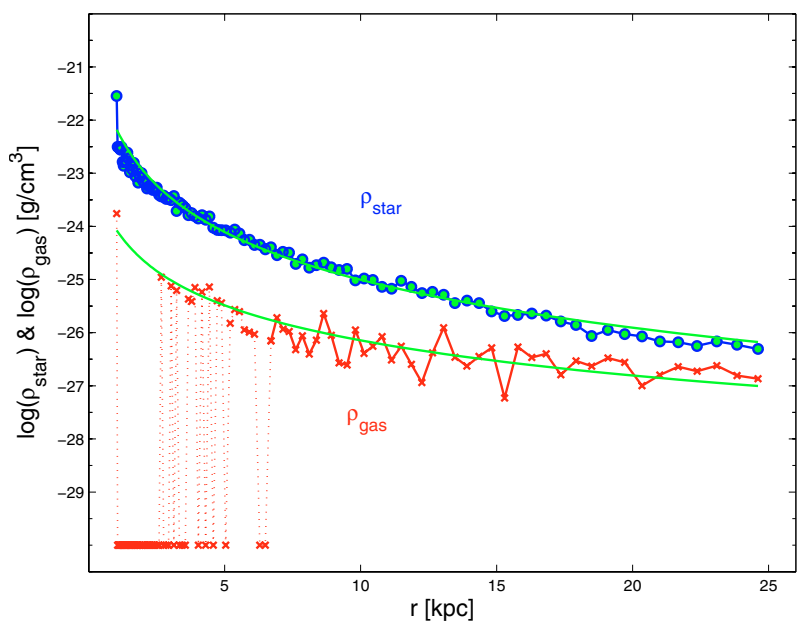

Fig. 6. The averaged density profiles of gas and stars for the model of $1.6 \times 10^{11} M_{\odot}$ baryonic mass at the age of $13 \mathrm{Gyr}$. The continuous lines are the best fit obtained.

In order to secure a smooth behaviour at the galaxy radius $R_{\mathrm{gal}}$, the star and gas density profiles are represented by the law:

$\rho_{i}=\rho_{0 i}\left[1+\left(\frac{r}{r_{\mathrm{c}}^{i}}\right)^{2}\right]^{-\gamma_{i}}$

where " $i$ " stands for "stars" or "gas", and $r_{\mathrm{c}}^{i}$ are the corresponding core radii. The above representation is suited more to our aims than is the classical King law. The fits are shown in Fig. 6. They are normalized in such a way that the integral over the galaxy volume corresponds to the amount of gas contained inside $R_{\text {gal }}$.

\subsubsection{Star formation rate}

In the dynamical models, the period of intense star formation, during which most of the star mass is built up, is confined within the first 3 to 4 Gyr. In Model A this is followed by a long tail of minimal stellar activity that continues forever. If this activity was real, we would expect a background of young stars giving rise to a significant emission in the UV-optical region up to the present, which is not compatible with the observed spectra of typical early-type galaxies. As already pointed out by Merlin \& Chiosi (2006) this minimal stellar activity is an artefact of the poor mass-resolution for the baryonic component, in other words, the low number of particles considered in the numerical simulations. To cope with this, we simply set the star formation rate to zero when only one or two star particles are involved. This is equivalent to cutting the star formation rate for ages older than about 5 Gyr. The problem does not occur with model B simply because the last computed model is at $6 \mathrm{Gyr}$.

\subsubsection{Checking dynamical models against chemo-spectro-photometric models}

To this aim we plugged the star formation history (SFH) of dynamical models into the chemical code of Portinari et al. (1998), and the closed-box approximation was adopted. The total baryonic mass of the chemical models is the same as in the dynamical ones, and equally so for the initial mass function of the stars composing each star particle: Kroupa (1998) in our case. In Fig. 7 we show the results obtained by inserting the SFH

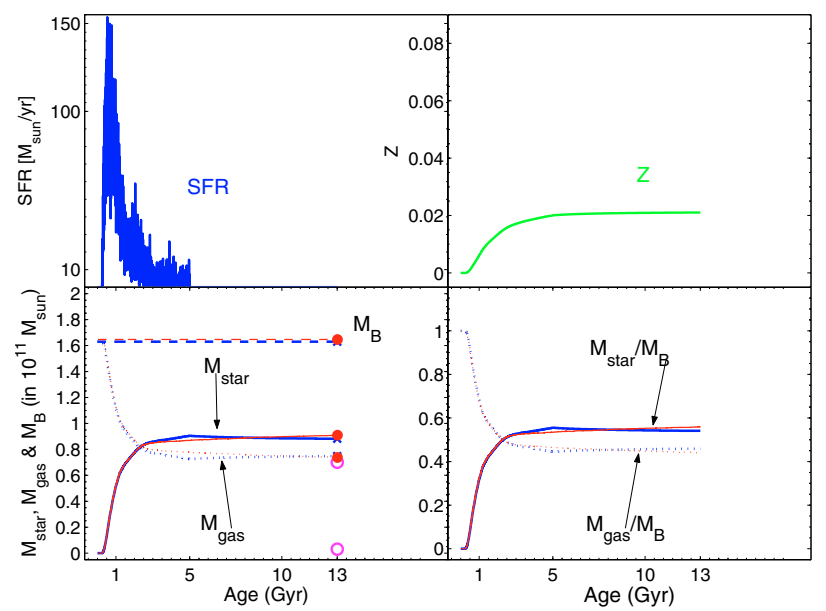

Fig. 7. Basic quantities of the chemical models for a prototype earlytype galaxy of $1.6 \times 10^{11} M_{\odot}$ baryonic mass as a function of the age. Topleft panel: the star formation rate obtained from the chemo-dynamical model of $1.6 \times 10^{11} M_{\odot}$ baryonic mass (SFR). Top-right panel: the evolution of the metallicity $Z$. Bottom-left panel: the masses for both the classical and the chemo-dynamical model of baryonic matter (thick and thin dashed lines), stars (thick and thin continuous lines), and gas (thick and thin dotted lines) are shown. The mass of gas and stars inside $25 \mathrm{kpc}$ are also shown (open circles) for the age of 13 Gyr. Bottom-right panel: the fractional masses for both the models of stars (thick and thin continuous lines) and gas (thick and thin dotted lines) are shown.

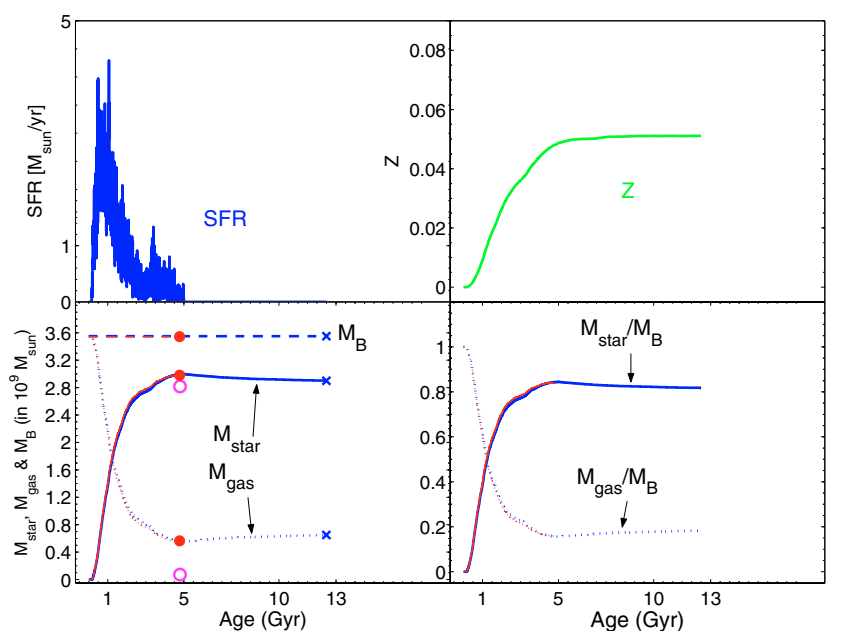

Fig. 8. The same as in Fig. 7, but for the model with total baryonic mass of $3.5 \times 10^{9} M_{\odot}$.

of Model A into a classical chemical model with total baryonic mass where $M_{\mathrm{B}}$ equal to $1.6 \times 10^{11} M_{\odot}$. The top panels display the adopted SFH (left) and the gas metallicity of the chemical model, respectively. The bottom left panel shows the temporal variation in the star mass $M_{\text {star }}$ and gas mass $M_{\text {gas }}$, whereas the bottom right panel shows the ratios $M_{\mathrm{star}} / M_{\mathrm{B}}$ and $M_{\mathrm{gas}} / M_{\mathrm{B}}$ for both the dynamical and chemical model. The agreement is very good, thus confirming the internal consistency between the descriptions of the same object. We also show the amount of gas at 13 Gyr contained in the whole galaxy for both the dynamical and the classical chemical models and the amount of gas contained inside $R_{\text {gal }}$ (open squares). Indeed there is little gas left over inside the $25 \mathrm{kpc}$ radius region. Similarly in Fig. 8, we show the results obtained inserting the SFH of Model B into a classical chemical model with total baryonic mass of $3.5 \times 10^{9} M_{\odot}$. The only difference is that the maximum age of the dynamical model 
is 5 Gyr. This cross-checking of the models is particularly significant because: first, it ensures that the results of the analytical models fairly reproduce those of the dynamical simulations as far as some important features are concerned; second, it ensures that we can safely use the result of chemical models to prolong the evolutionary history of Model B up to the present; third, we can safely apply the population synthesis technique of Piovan et al. (2006b).

Knowing the amount of gas, we need to specify the fraction of it in the form of dust to finally be able to derive the whole SED from X to FIR and look for relationships between the luminosity in the X, B, and FIR pass-bands we want to interpret. Our models, both semi-analytical and chemo-dynamical, are not suitable to describing the evolution of the compositions and abundances of both gas and dust phases. The relative proportions of the various components of the dust would require detailed study of the evolution of the dusty environment and complete information on the dust yields, as in the models of Dwek (1998, 2005). This would lead to a better and more physically sounded correlation between the composition of dust and the star formation and chemical enrichment history of the galaxy itself, although at the price of increasing the complexity and the uncertainty of the problem.

The key parameter in calculating the amount of dust is the dust-to-gas ratio, defined as $\delta=M_{\mathrm{d}} / M_{\mathrm{H}}$, where $M_{\mathrm{d}}$ and $M_{\mathrm{H}}$ are the total dust and hydrogen mass, respectively. For the Milky Way and the galaxies of the Local Group, $\delta$ is estimated to vary from about $1 / 100$ to $1 / 500$ and typical values $\delta=0.01$, $\delta=0.00288$, and $\delta=0.00184$ are used for the Milky Way (MW) and the Large and Small Magellanic Clouds (LMC and SMC). These dust-to-gas mass ratios describe a decreasing sequence, going from the MW to the LMC and SMC. Since these galaxies also describe a sequence of decreasing metallicity, a simple assumption is to hypothesize $\delta \propto Z$ in such a way as to match the approximate results for MW, LMC, and SMC: $\delta=\delta_{\odot}\left(Z / Z_{\odot}\right)$. This relation simply implies that the higher the metal content of a galaxy, the higher the abundance of grains per $H$ atom. However, the metallicity difference not only implies a difference in the absolute abundance of heavy elements in the dust, but also a difference in the composition pattern as a function of the star formation history (Dwek 1998, 2005). Despite these uncertainties (Devriendt et al. 1999), the relation $\delta \propto Z$ is often adopted to evaluate the amount of dust in galaxy models (e.g. Silva et al. 1998) by simply scaling the dust content adopted for the ISM of the MW to the metallicity under consideration.

The $1.6 \times 10^{11} M_{\odot}$ and $3.5 \times 10^{9} M_{\odot}$ galaxy models reach an average metallicity of solar and slightly more than twice solar, respectively. To describe them, we adopted the description of Piovan et al. (2006a,b) where a model of dusty ISM is built by taking different metallicities into account. The problem, however, remained unsettled for metallicities higher than the solar one, where relative proportions holding good for the MW average diffuse ISM model were adopted and the amount of dust scaled with $\delta \propto Z$. Therefore, for the $1.6 \times 10^{11} M_{\odot}$ galaxy with solar metallicity, the MW diffuse-ISM model was adopted $\left(\delta=\delta_{\odot}\right)$, while for the $3.5 \times 10^{9} M_{\odot}$ model we followed the $\delta \propto Z$ relation, using the MW average pattern of dust composition.

The connection between the results of this model and the observed diagrams are discussed in the next section.

\section{Discussion}

Our data confirm and extend the previous relations between various tracers of the ISM in galaxies of different morphological types. In the literature the relation found by Bregman et al. (1992) between $S_{\text {CO }}$ and $S_{100}$ indicates a direct proportionality (slope $=1$ ) between the two fluxes and differs from that of Solomon \& Sage (1988), which exhibits a steeper gradient. Our relation (1) agrees quite well with the proportionality found by Bregman et al. (1992), the slope we found being equal to 1.06. The similarity between the two curves in Fig. 1 is evident. As described in the introduction, this link derives from the excitation of gas clouds by the currently forming stars and by the warming of the dust in the galaxy.

\subsection{Late-type galaxies}

In late-type galaxies $(t>\mathrm{Sb})$ our data show the existence of a linear relation between soft X-ray fluxes and other indicators of recent and current star formation, such as the $B$ and FIR luminosity, respectively (Eqs. (4) and (7)). This has been known since the first X-ray observations of large samples Fabbiano et al. (1992), and this connection between $B$ and X-ray luminosity in late type galaxies has been interpreted as due to the contribution of discrete X-ray sources, whose number is proportional to the quantity of already formed stars (Ciotti et al. 1991; Beuing et al. 1999). The recent work of Kim \& Fabbiano (2004), which is able to resolve the single X-ray binaries in 14 galaxies, indicates that the X-ray luminosity produced by discrete sources is related to $\mathrm{B}$ luminosity by a similar relation, with an intercept value of -3.63 , similar to our -3.85 of Eq. (4).

In addition to the interstellar radiation, which is proportional to the number of already formed stars, the X-ray emission is also produced by HII regions, where there is an ongoing vigorous star formation (David et al. 1992). This latter contribution appears more evident in FIR light and may explain the existence of a similar linear relation between $L_{\mathrm{X}}$ and $L_{\mathrm{FIR}}$.

\subsection{Early-type galaxies}

In early type galaxies the behaviour of these relations is quite different. For most of these galaxies, the star formation is exhausted and it may be present in a few of them, eventually fed by gas accretion phenomena. Different mechanisms have been suggested to explain the X-ray emission in this kind of galaxies. In particular the main ones are the thermal emission due to hot ISM and the emission generated by a relatively old population of end objects of stellar evolution, composed of type I supernovae remnants and low-mass X-ray binaries that have not yet evolved. In particular, the X-ray emission for the fainter galaxies is compatible with discrete sources and seems to be dominated by compact accreting systems, while for the brighter objects the emission from hot diffuse gas still present in the galactic-potential well is present as an additional component (Beuing et al. 1999). The number size of this population of relatively old objects is well represented by the total blue luminosity of the galaxy. For this reason the X-ray fluxes are still linked in early type galaxies to the total blue luminosity, representing the more recent part of the history of star formation in the galaxy.

In the FIR, however, mechanisms different from the emission from warm dust heated by the newly born stars predominate, since the star formation in most of these systems is almost exhausted. The FIR emission comes from circumstellar dusty shells around AGB stars and from an interstellar medium due to the outflow of dusty gas from AGB and RGB stars, as described in Sect. 4. 


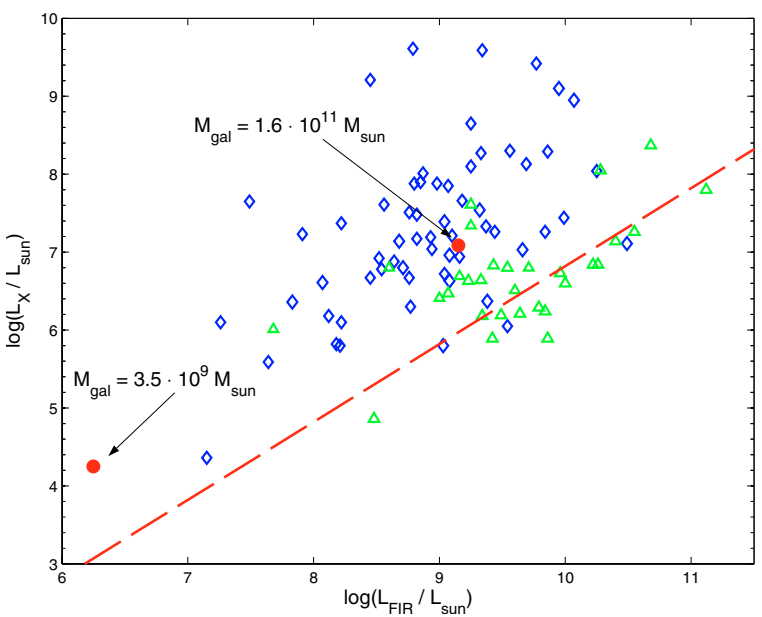

Fig. 9. $L_{\mathrm{FIR}}$ vs. $L_{\mathrm{X}}$ diagram as in Fig. 3, right panel, but with the values deduced for the two template models of the early-type galaxy (filled circles). The diamonds represent the $E-S 0$ galaxies, the triangles the $S 0 a-S b$ galaxies. The masses of the two theoretical models are also indicated.

The key point for interpreting the observed trends is that we are dealing with an emission coming from a more or less small amount of dust distributed over the whole galaxy and heated by an average interstellar radiation field due to all the stars of any age. The situation is quite different from what happens, for instance, in starburst galaxies where high optical depth dusty regions reprocess the light coming from newly born stars embedded in the parental environment. We can therefore conclude that in most of our early-type galaxies, the mechanism of IR emission is not strictly related to the star formation, and the link between the younger generations of stars and dust emission is lost. For these reasons one may expect that the soft X-ray luminosity in early type galaxies is traced by the total blue luminosity but not by the FIR luminosity. With the end of the star formation, the FIR emission of these galaxies has faded out and an early type galaxy with the same $L_{\mathrm{X}}$ of a late type will have a lower $L_{\mathrm{FIR}}$. This could explain the location of the points in Fig. 3 (right panel), on the left side of the linear relation.

To check if this interpretation is correct we try to apply the detailed chemo-dynamical spectrophotometric model described in the previous section, in such a way as to estimate the luminosities produced by the stars in connection with the various phenomena present inside the galaxy, taking the contribution by dust into account as well. Since the theoretical model cannot derive the $L_{X}$ luminosity, we proceed in the following way.

The luminosities $L_{B}$ and $L_{\mathrm{FIR}}$ are directly derived from the model. Then, we assume that the X-ray production of these galaxies is proportional to $L_{B}$ according to our relation (10). In this way we may estimate the expected X-ray flux and define a representative point in the $L_{\mathrm{FIR}}$ vs. $L_{\mathrm{X}}$ plot.

We start considering two template models in which all the parameters are fixed using the clues coming from the dynamical simulations of Merlin \& Chiosi (2006), as described in Sect. 4. The King profiles represented in Fig. 6 are similar for all the components, with $\gamma_{\text {stars }} \simeq \gamma_{\text {gas }} \simeq 1.5$ and $r_{\mathrm{c}}^{\text {stars }} \simeq r_{\mathrm{c}}^{\text {gas }} \simeq 0.5 \mathrm{kpc}$, while the dimension of the galaxy is an average one corresponding to most of the galaxies available in the catalogue. The SFH is exactly the one obtained by the dynamical simulations. The two values of $L_{\mathrm{FIR}}$ and $L_{\mathrm{X}}$ obtained for the $3.5 \times 10^{9} M_{\odot}$ and $1.6 \times 10^{11} M_{\odot}$ baryonic mass models are plotted in Fig. 9 . The more massive galaxy fits well into the region defined by the

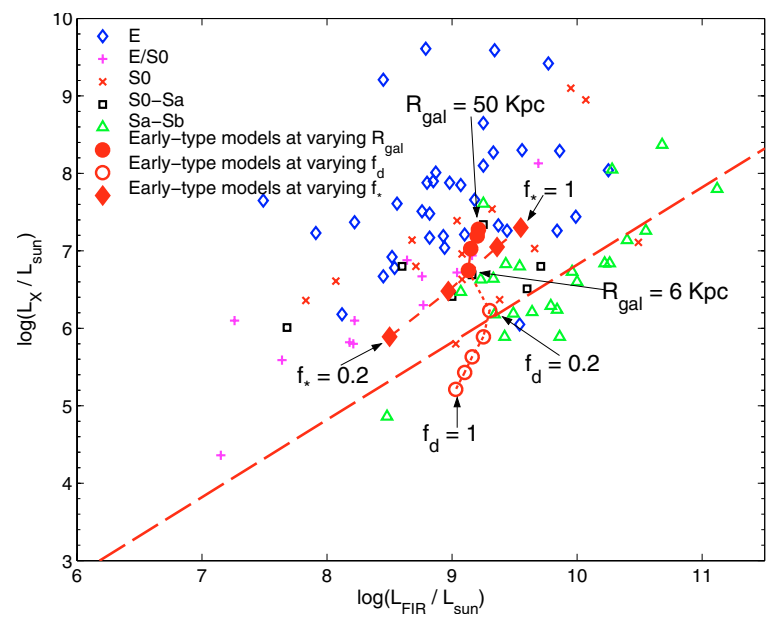

Fig. 10. Models of the $1.6 \times 10^{11} M_{\odot}$ baryonic mass galaxy in the $L_{\mathrm{FIR}}$ vs. $L_{\mathrm{X}}$ diagram with the variation of the galactic radius (filled circles), the mass of stars (filled diamonds), and finally, the mass of gas (empty circles). We represented: $E$ galaxies (empty diamonds), $E / S O$ galaxies (pluses), $S 0$ galaxies (crosses), $S 0 s$ galaxies (empty squares), $S a-S b$ (empty triangles).

actually observed galaxies. The calculated levels of emission $L_{\mathrm{X}}$ and $L_{\mathrm{FIR}}$ of this galaxy are very low, and for this reason they belong to a region where we do not have enough observations. Its weak $L_{\mathrm{FIR}}$ emission can be explained by the dynamical evolution in which almost all the gas is consumed to form stars so the galactic winds are very efficient (see Chiosi \& Carraro 2002, for more details about galactic winds in low-mass galaxies). Therefore, even if the trend of this galaxy is the expected one for early-type galaxies (the model stays above the linear relation), nothing can be said with more certainty, because we lack observed data in that region of the diagram.

Much more interesting is the model of higher mass. The calculated luminosities of the model, with its exhaustion of its star formation, seem to agree well with the observations of early-type galaxies. However, the model needs to be checked against other possibilities, in order to understand how the various parameters of the model influence the spreading of early-type galaxies into the observational data. First of all we have to check the effects of the geometrical parameters and of the masses of stars/gas.

\subsubsection{The galactic radius}

In Fig. 10 we show the model of $1.6 \times 10^{11} M_{\odot}$ baryonic mass when varying the galactic radius, keeping the galactic centre in the centre of mass of the stellar component. The radii taken into account range from $6 \mathrm{kpc}$ to $50 \mathrm{kpc}$. All the other parameters are fixed. Four models are represented and connected by a continuous line. For larger radii we observe an increase in both $L_{\mathrm{FIR}}$ and $L_{\mathrm{X}}$, with a more emphasized increase in $L_{\mathrm{X}}$. Since the density profile is unchanged, both the increases in luminosity are simply due to the bigger amount of material considered, taking larger radii in the dynamical simulation into account. The stronger increase in $L_{\mathrm{X}}$ than $L_{\mathrm{FIR}}$ can be explained simply: $L_{\mathrm{X}}$ is linearly related to $L_{B}$, which is directly connected to the stellar luminosity. The stellar component is more massive and more concentrated toward the centre than the gaseous one (Fig. 5, upper panel). It follows that at increasing radius we introduce more stars and more gas into the models, but the added amount of stars is bigger than the gaseous one, shifting $L_{B}$ (and then the linearly related $L_{\mathrm{X}}$ ) more than $L_{\mathrm{FIR}}$. Finally, we observe how, even if 
taking the smallest radius of $6 \mathrm{kpc}$ into account, it is not possible to move the theoretical point near the linear relation holding for spirals.

\subsubsection{The masses of stars and gas}

We also investigated in Fig. 10 what happens if we forget about the clues coming from dynamical simulations on the masses of stars and gas, and we arbitrarily start varying the amounts of stars or gas, keeping everything fixed. The shift of the model of lowest radius if we are changing the mass of stars inside $R_{\mathrm{gal}}$, going in fractions from $f_{*}=0.2$ to $f_{*}=1.0$ of the total amount of stars in the dynamical model. The effect is simply to move the point along a line about parallel to the linear relation. A smaller amount of stars implies a lower luminosity $L_{B}$ (and therefore a lower $L_{X}$ ), but also a lower $L_{\mathrm{FIR}}$, because the weaker radiation field makes dust cooler and shifts the peak of dust emission to wavelengths longer than $100 \mu \mathrm{m}$, resulting in smaller $L_{\mathrm{FIR}}$. Finally, we show five models in Fig. 10 by obtained fixing the amount of stars and varying the mass of gas (and therefore of dust) from $f_{\mathrm{d}}=0.2$ to $f_{\mathrm{d}}=1.0$, of the total amount of gas in the dynamical model.

The effect of this huge increase in the mass of diffuse gas and dust (in the original model at $R_{\mathrm{gal}}=6 \mathrm{kpc}$ only $0.03 \%$ of the gas is inside $R_{\text {gal }}$ ) is to shift the models straight toward the linear relation. It can be explained in the following way. Increasing the amount of diffuse gas/dust (with all the parameters fixed and the star formation exhausted) implies more absorption of the stellar radiation and therefore a lower $L_{B}$ (and $L_{X}$ ). On the other hand, $L_{\mathrm{FIR}}$ remains almost unchanged or becomes lower. The reason is that the strongly increased mass of dust makes the average stellar radiation field weaker, therefore the increased emission of dust (due to the bigger mass) peaks at wavelengths longer than $100 \mu \mathrm{m}$, leaving $L_{\mathrm{FIR}}$ almost unchanged. Even if in this way we can shift the model toward the linear relation, the situation is physically unrealistic, requiring a huge amount of gas/dust concentrated in the centre of an early-type galaxy with exhausted star formation, which is not commonly observed and also not predicted by dynamical models.

\subsubsection{The scale radii}

Other geometrical parameters that must be examined are the scale radii $r_{\mathrm{c}}^{i}$ of the King's laws - Eq. (12) - that describe the distribution of the stellar and gaseous components. The averaged profiles shown in Fig. 6 and used for the models of Figs. 9 and 10 are both characterised by $r_{\mathrm{c}}^{i} \simeq 0.5$, allowing for a concentrated amount of stars and gas in the inner regions. Keeping all the other parameters fixed, we investigated what happens if we allow for a uniform distribution of one or both of the physical components. Three cases have been considered: a uniform distribution of gas keeping the stellar one fixed $\left(r_{\mathrm{c}}^{\text {gas }} \rightarrow \infty, r_{\mathrm{c}}^{\text {stars }} \simeq 0.5\right)$, a uniform distribution of stars keeping fixed the gaseous one $\left(r_{\mathrm{c}}^{\text {stars }} \rightarrow \infty, r_{\mathrm{c}}^{\text {gas }} \simeq 0.5\right)$ and, finally, a uniform distribution of both the components $\left(r_{\mathrm{c}}^{\text {stars }} \rightarrow \infty, r_{\mathrm{c}}^{\mathrm{gas}} \simeq \infty\right)$. The results are shown in Fig. 11 for two radii of the galaxy model, $R_{\text {gal }}=6 \mathrm{kpc}$ and $R_{\text {gal }}=20 \mathrm{kpc}$, respectively. The three different distributions give a similar result: a lower $L_{\mathrm{FIR}}$, shifting the point to the left, and a slightly higher $L_{\mathrm{X}}$.

This can be explained in the following way: for $r_{\mathrm{c}}^{\text {stars }}$ and $r_{\mathrm{c}}^{\text {gas }}$ both $\simeq 0.5$ and the diffuse ISM and the stars are both concentrated in the inner region of the galaxy with a density of stars/gas of

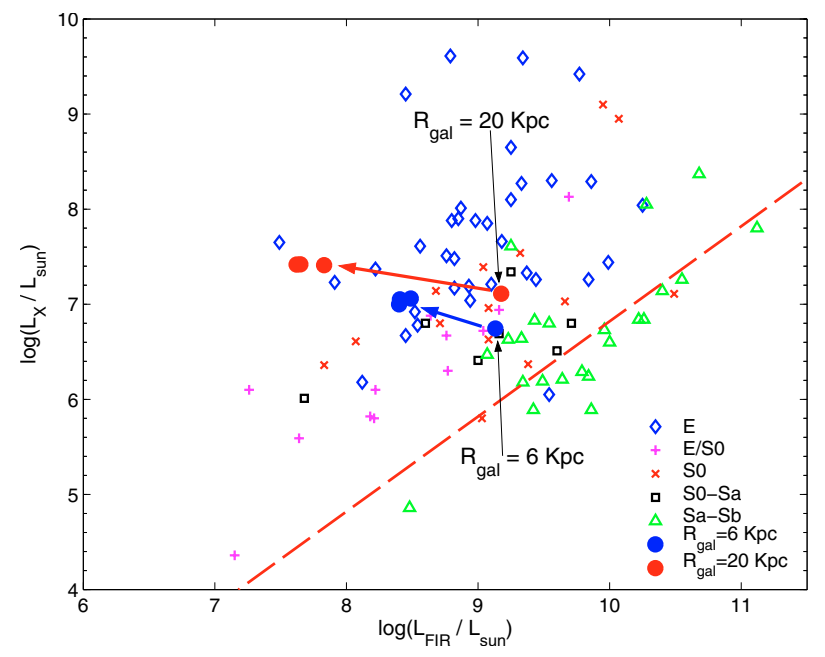

Fig. 11. Models of the more massive galaxy of $1.6 \times 10^{11} M_{\odot}$ baryonic mass in the $L_{\mathrm{FIR}}$ vs. $L_{\mathrm{X}}$ diagram for various core radii $r_{\mathrm{c}}^{\text {stars }}$ and $r_{\mathrm{c}}^{\text {gas }}$, plotted as filled circles. The thin arrows indicate the original model, while the thick arrows indicate the shift of the model for various core radii. We represented: $E$ galaxies (empty diamonds), $E / S 0$ galaxies (pluses), $S 0$ galaxies (crosses), $S 0 s$ galaxies (empty squares), $S a-S b$ (empty triangles).

many orders of magnitude higher than the outer regions. This is the condition for producing high $L_{\mathrm{FIR}}$, because we find that the regions of higher density of dust are the same in which there is also the higher average radiation field heating dust. The spatial distribution of the ISM favours the interaction with the stellar radiation. When we destroy this coupling between stellar emission and density of gas, as we do allowing for a uniform distribution of gas or stars or both, the emission in the $L_{\mathrm{FIR}}$ becomes weaker. The weakening of the dusty emission is stronger for the bigger radius of $20 \mathrm{kpc}$ because in all three cases one or both the components are distributed over a huge galactic volume and we then have a low density of gas, eventually coupled with weak radiation field. For the $6 \mathrm{kpc}$ model, even if the coupling in the central regions is destroyed, the galaxy is small enough to keep a acceptable level of $L_{\text {FIR }}$, even when the matter is equally distributed across all the galaxy volume.

\subsubsection{The star formation history}

The last and main point to be examined is how varying the star formation history affects the position of the galaxies on the $L_{\mathrm{FIR}}$ vs. $L_{X}$ plot. In Figs. 10 and 11, the galaxies of different morphological types form a sequence that, going from systems in which the star formation was exhausted long ago to systems in which star formation is still active, moves toward the linear relation and thereby suggesting the key role played by star formation. First of all we calculate the $L_{\mathrm{FIR}}$ and $L_{\mathrm{X}}$ obtained by the SEDs and the models by Piovan et al. (2006b) of real galaxies of the local universe: three spiral galaxies (M 100, M 51 and NGC 6946) and two starburst galaxies (Arp 220 and M 82). The key point is that the SFHs of these galaxies allow us to cover an acceptable number of different star formation histories. All these SFHs, unlike the ones of the ellipticals obtained by dynamical simulations, never end; in the case of the two starbursters, a strong burst of star formation is added in the last millions of years. A huge amount of $L_{\mathrm{FIR}}$ therefore comes from the young and deeply obscured region of star formation and not only from the diffuse component. 


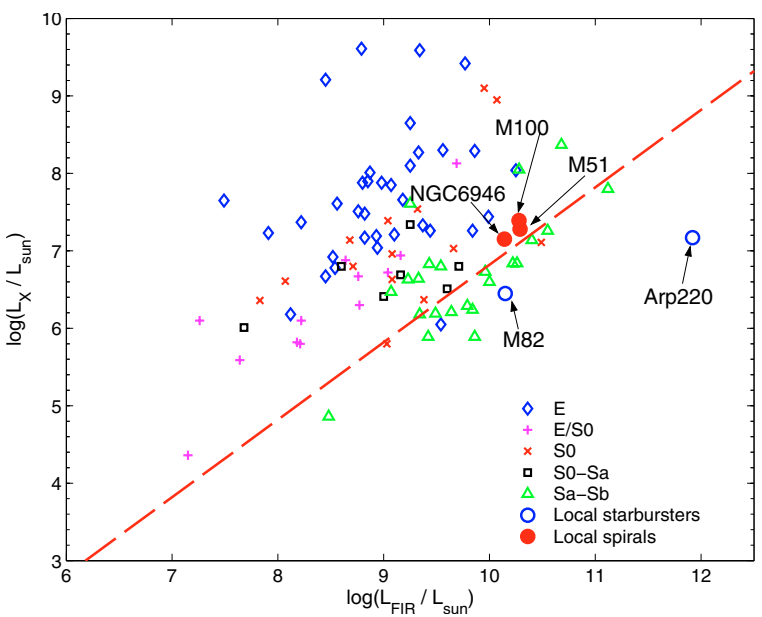

Fig. 12. $L_{\mathrm{FIR}}$ vs. $L_{\mathrm{X}}$ for galaxy models of spirals and starbursters. Five theoretical models, taken from Piovan et al. (2006b), are represented: three spirals (M 100, M 51 and NGC 6946) and two starbursters (Arp 220 and M 82). Different morphological types are plotted as in Fig. 11.

The results, presented in Fig. 12, show that the three models of spirals stay near the linear relation, while the two starbursters stay below the line, with the model of Arp 220, powered by a huge burst of star formation falling well below the linear relation. The stronger the emission coming from the regions of star formation, the bigger the shift toward higher $L_{\mathrm{FIR}}$ and lower $L_{X}$ (due to the lower $L_{B}$ ). The results from these models are quite similar to the observational data: for M 100 we get $\left(L_{\mathrm{FIR}}, L_{\mathrm{X}}\right)=(10.28,7.29)$ with the observations giving $(10.37,7.01)$, for Arp 220 we have $\left(L_{\mathrm{FIR}}, L_{\mathrm{X}}\right)=(11.92,7.17)$ compared with $(11.99,7.60)$, and for $\mathrm{M} 82$ we get $\left(L_{\mathrm{FIR}}, L_{\mathrm{X}}\right)=$ $(10.15,6.45)$ against $(9.79,6.31)$. However, these galaxy models, even if they represent real galaxies well, differ in many parameters from the early-type galaxy model of $1.6 \times 10^{11} M_{\odot}$, like geometry and mass. These parameters, together with the SFH, obviously concur to determine the position of the models on the $L_{\mathrm{FIR}}$ vs. $L_{\mathrm{X}}$ plot. To isolate the effect of the $\mathrm{SFH}$, we first re-calculated the SFHs of the five theoretical models above, rescaled to the mass of $1.6 \times 10^{11} M_{\odot}$ of the early-type galaxy model. In Fig. 13 we can see four of the five SFH obtained. Second, we fixed all the geometrical parameters to the same values as for the average model of the $1.6 \times 10^{11} M_{\odot}$ early type galaxy. The additional parameters, i.e. the escape time of young stars from parental molecular clouds, the library of SEDs of young dusty regions and the mass of gas in the diffuse and molecular component, are fixed to the values used in Piovan et al. (2006b) for spirals and starbursters, as appropriate.

In Fig. 14 we finally show the results obtained as a function of the SFH for the galaxy of $1.6 \times 10^{11} M_{\odot}$, keeping all the other parameters fixed. It is interesting to observe that, since now the star formation never ends and the galactic wind is not included, the classical semi-analytical chemical evolution can be much more safely coupled to the spectro-photometric code. The effect of varying the SFH at fixed mass is to enhance the $L_{\mathrm{FIR}}$, keeping the $L_{\mathrm{X}}$ almost fixed and shifting the points toward the linear relation at higher infrared luminosities. This is ultimately due to the strong and efficient reprocessing of the light coming from very young stars, occurring in the dusty star-forming regions. As a consequence of this, models with starburst-like SFHs shift, as expected, toward higher $L_{\mathrm{FIR}}$ luminosity than do models with spiral-like SFH, because of the stronger star
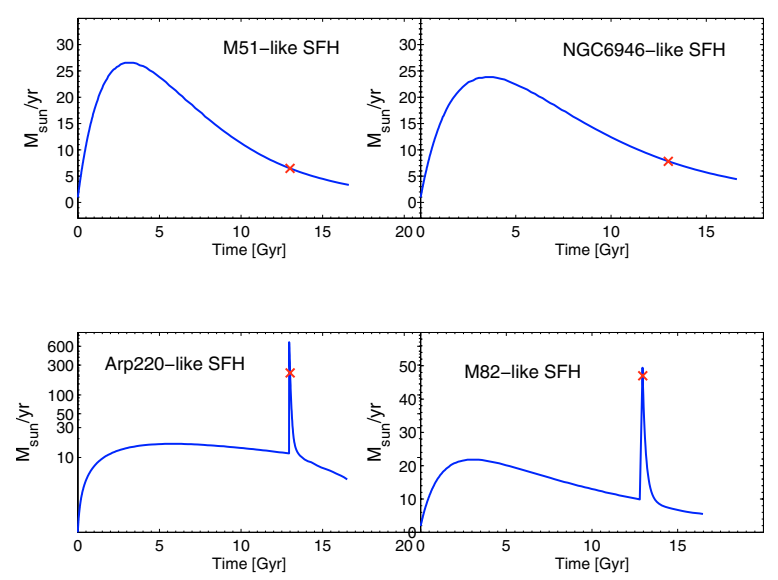

Fig. 13. Different adopted star formation histories for the model of $1.6 \times$ $10^{11} M_{\odot}$ baryonic mass. The SFHs of Piovan et al. (2006b) have been re-calculated for the same mass of the dynamical model. The upper panels represent two spiral-like SFHs, while lower panels show two starburst-like SFHs that peaked in the last million years.

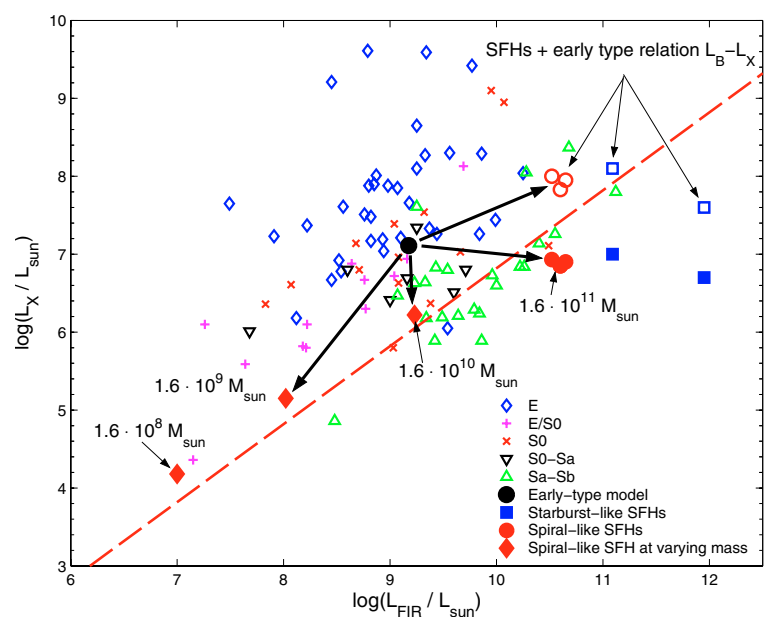

Fig. 14. $L_{\mathrm{FIR}}$ vs. $L_{\mathrm{X}}$ for galaxy models of fixed $1.6 \times 10^{11} M_{\odot}$ baryonic mass for various SFHs (large circles and squares) or at fixed SFH for various masses from $1.6 \times 10^{8} M_{\odot}$ to $1.6 \times 10^{11} M_{\odot}$ (large filled diamonds). Large circles and squares represent, respectively, models with spiral-like SFH and starburst-like SFH. Large filled circles and squares are obtained using Eq. (4), while large open circles and squares depend on Eq. (10). Different morphological types are plotted as in Fig. 11.

formation and, therefore, emission from young dusty regions. This can be also understood if we look in detail at the relative contribution to $L_{\mathrm{FIR}}$ coming from the regions of star formation (let us define it $\left.f_{\mathrm{MCs}}\right)$ and represent it as usual in $\log \left(L_{\mathrm{FIR}} / L_{\odot}\right)$ and from the diffuse interstellar medium $\left(f_{\mathrm{ISM}}\right)$. We get the values $\left(f_{\mathrm{SFR}}=10.15, f_{\mathrm{ISM}}=10.48\right),\left(f_{\mathrm{SFR}}=10.02, f_{\mathrm{ISM}}=10.45\right)$, $\left(f_{\mathrm{SFR}}=9.98, f_{\mathrm{ISM}}=10.37\right)$ for the three models with spirallike SFHs, while we have $\left(f_{\mathrm{SFR}}=10.97, f_{\mathrm{ISM}}=10.47\right)$ and $\left(f_{\mathrm{SFR}}=11.93, f_{\mathrm{ISM}}=10.56\right)$ for the models with starburst-like SFHs. The stronger the contribution from star forming regions, the higher is $L_{\mathrm{FIR}}$ when keeping $L_{B}$ (and $L_{\mathrm{X}}$ ) almost unchanged. Models slightly dominated by the ISM contribution, but with a significant contribution coming from obscured newly born stars are more suitable to the linear relation of spirals.

It is worth noticing that in Fig. 14 we show both the results obtained by applying the early-type linear relation between $L_{X}$ and $L_{B}-$ Eq. (10) - and the late-type one - Eq. (4). Since, however, the SFHs used (see Fig. 13) are typical of late type galaxies 
(or starbursters), it is physically much sounder to apply Eq. (4) to obtain the $L_{\mathrm{X}}$ luminosity. As a last point, we also calculated a sequence of models in which one of the SFHs of the spirals has been chosen (namely the one of NGC 6946) with all the parameters fixed and only the mass varied. As we see from Fig. 14, the effect of varying the mass is to shift the object diagonal by almost along the relation. This is explained simply by the smaller amounts of stars/gas emitting radiation.

\section{Conclusions}

We have been able to describe the relations existing in a galaxy between the various tracers of the ISM and to fix the coefficients of the relations existing between FIR, $B$, and X-ray luminosity, both for early- and late-type galaxies.

The large set of data we used allowed us to more clearly redefine the relationship between the $\mathrm{CO}$ and the $100 \mu$ m fluxes. We found that the relation, first obtained by Bregman et al. (1992) for early type galaxies, is also valid for late type galaxies. In these galaxies, the X-ray flux also appears linked to $B$ and FIR emissions.

The only relation lacking from observations, i.e. the one between $L_{\mathrm{X}}$ and $L_{\mathrm{FIR}}$, was studied by the use of the most recent chemo-dynamical models coupled with dusty evolutionary population synthesis.

The calculated luminosities of the models seem to confirm our hypothesis about a connection between the exhaustion of the star formation and the "migration" of the early type galaxies above the linear relation in the $L_{\mathrm{X}}$ vs. $L_{\mathrm{FIR}}$ plot. In the frame of our assumptions, we may therefore conclude that the prediction of our dusty chemo-dynamical models of galaxy evolution is consistent with the observed lack of a direct relation between $L_{\mathrm{X}}$ and $L_{\mathrm{FIR}}$ for early type galaxies and is due to the different mechanisms for producing FIR light in galaxies where the active star formation is no longer active. In most of our early-type galaxies, the mechanism of IR emission is no longer strictly related to the ongoing star formation and to the reprocessing of the radiation in the dense regions where new stars are born. The FIR emission therefore comes most likely from circumstellar dusty shells around AGB stars and from an interstellar diffuse medium due to the outflow of dusty gas from AGB and RGB stars.

Finally, we can summarize that: (i) the SFH of the galaxies seems to have the stronger effect on the position of early-type galaxies in the $L_{\mathrm{X}}$ vs. $L_{\mathrm{FIR}}$ plot; (ii) other parameters, like the radius of the galaxy and the scale radii of stars and gas, play a secondary role, even if they can significantly contribute to the scatter of the models in the region above the linear relation; (iii) the mass is the main parameter in explaining the scatter of the points along the linear relation.

Acknowledgements. This research was partially funded by the University of Padua with Funds ex 60\% 2005. We acknowledge Prof. C. Chiosi for useful discussions of the theoretical subjects of this paper. L. Piovan is pleased to acknowledge the hospitality and stimulating environment provided by the MaxPlanck-Institut für Astrophysik in Garching where part of the work was done during his visit as an EARA fellow on leave from the Department of Astronomy of the Padua University. We also thank the referee for the detailed and useful comments about this topic.

\section{References}

Arimoto, N., \& Yoshii, Y. 1987, A\&A, 173, 23 Arimoto, N., \& Yoshii, Y. 1989, A\&A, 224, 361
Arimoto, N., \& Tarrab, I. 1990, A\&A, 228, 6

Arp, H. 1966, Atlas of Peculiar Galaxies Publisher: California Institute of Technology, Pasaadena, CA

Arp, H. C., \& Madore, B. F. 1987, A Catalog of Southern Peculiar Galaxies and Associations (Cambridge University Press)

Bettoni, D., Galletta, G., \& García-Burillo, S. 2003a, A\&A, 405, 5

Bettoni, D., Galletta, G., \& Garcia-Burillo, S. 2003b, VizieR Online Data Catalog, 340, 50005

Beuing, J., Döbereiner, S., Böhringer, H., \& Bender, R. 1999, MNRAS, 302, 209 Bregman, J. N., Hogg, D. E., \& Roberts, M. S. 1992, ApJ, 387, 484

Bressan, A., Chiosi, C., \& Fagotto, F. 1994, ApJS, 94, 63

Bressan, A., Chiosi, C., \& Tantalo, R. 1996, A\&A, 311, 425

Bruzual, G, \& Charlot, S. 1993, ApJ, 405, 538

Buzzoni, A. 2002, AJ, 123, 1188

Buzzoni, A. 2005, MNRAS, 361, 725

Casasola, V., Bettoni, D., \& Galletta, G. 2004a, A\&A, 422, 941

Casasola, V., Bettoni, D., \& Galletta, G. 2004b, VizieR Online Data Catalog, 342, 20941

Carraro, G., Lia, C, \& Chiosi, C., 1998, MNRAS, 297, 1021

Chiosi, C. 1980, A\&A, 83, 206

Chiosi, C., Bressan, A., Portinari, L., \& Tantalo, R. 1998, A\&A, 339, 355

Chiosi, C. 2000, A\&A, 364, 423

Chiosi, C., \& Carraro, G. 2002, MNRAS, 335, 335

Ciotti, L., Pellegrini, S., Renzini, A., \& D'Ercole, A. 1991, ApJ, 376, 380

David, L. P., Jones, C., \& Forman, W. 1992, ApJ, 388, 82

De Lucia, G., Springel, V., White, S. D. M., \& Kauffmann, G. 2006, MNRAS, 366,499

de Vaucouleurs, G., de Vaucouleurs, A., Corwin, H. G., et al. 1991, Third Reference Catalogue of Bright Galaxies (RC3) (New York: Springer-Verlag) Devereux, N. A., \& Young, J. S. 1991, ApJ, 371, 515

Devriendt, J. E. G., Guiderdoni, B., \& Sadat, R. 1999, A\&A, 350, 381

Dwek, E. 1998, ApJ, 501, 643

Dwek, E. 2005, The Spectral Energy Distributions of Gas-Rich Galaxies: Confronting Models with Data, ed. C. C. Popescu, \& R. J. Tuffs, AIP Conf., Proc., 761, 103

Fabbiano, G., Kim, D.-W., \& Trinchieri, G. 1992, ApJS, 80, 531

Gibson, B. K., \& Matteucci, F. 1997a, ApJ, 475, 47

Gibson, B. K. 1997b, MNRAS, 290, 471

Griffiths, R. E., \& Padovani, P. 1990, ApJ, 360, 483

Guhathakurta, P., Knapp, G. R., Kim, D. W., \& Jura, M. 1986, Baas, 18, 926

Ho, L. C., Filippenko, A. V., \& Sargent, W. L. W. 1997, ApJS, 112, 315

Kawata, D. 2001, ApJ, 558, 598

Kim, D.-W., \& Fabbiano, G. 2004, ApJ, 611, 846

Kodama, T., \& Arimoto, N. 1997, A\&A, 320, 41

Knapp, G. R., Guhathakurta, P., Kim, D.-W., \& Jura, M. A. 1989, ApJS, 70, 329 Kuntschner, H. 2000, MNRAS, 315, 184

Kroupa, P. 1998, MNRAS, 298, 231

Larson, R. B. 1974, MNRAS, 169, 229

Larson, R. B., \& Dinerstein, H. L. 1975, PASP, 87, 911

Merlin, E., \& Chiosi, C. 2006, MNRAS, in press [arXiv: astro-ph/0605052]

Paturel, G., Andernach, H., Bottinelli, L., et al. 1997, A\&AS, 124, 109

Piovan, L., Tantalo, R., \& Chiosi, C. 2006a, MNRAS, 366, 923

Piovan, L., Tantalo, R., \& Chiosi, C., 2006b, MNRAS, in press [arXiv: astro-ph/0605541]

Popescu, C. C., Tuffs, R. J., Völk, H. J., Pierini, D., \& Madore, B. F. 2002, ApJ, 567,221

Portinari, L., Chiosi, C., \& Bressan, A. 1998, A\&A, 334, 505

Ranalli, P., Comastri, A., \& Setti, G. 2003, A\&A, 399, 39

Sanders, D. B., \& Mirabel, I. F. 1985, ApJ, 298, L31

Sanders, D. B., Scoville, N. Z., Young, J. S., et al. 1986, ApJL, 305, L45

Silva, L., Granato, G. L., Bressan, A., \& Danese, L. 1998, ApJ, 509, 103

Solomon, P. M., \& Sage, L. J. 1988, ApJ, 334, 613

Springel, V., Yoshida, N., \& White, S. D. M. 2001, New Astron., 6, 79

Tagaki, T., Vansevicius, V., \& Arimoto, N. 2003, Publ. Astron. Soc. Jpn., 55, 385

Tantalo, R., Chiosi, C., \& Bressan, A. 1996, A\&A, 311, 361

Tantalo, R., Chiosi, C., Bressan, A., Marigo, P., \& Portinari, L. 1998, A\&A, 335, 823

Tantalo, R., \& Chiosi, C. 2004, MNRAS, 353, 405

Thomas, D., Maraston, C., Bender, R., \& de Oliveira, C. M., 2005, ApJ, 621, 673

Thronson, H. A., \& Telesco, C. M. 1986, ApJ, 311, 98

Trager, S. C., Faber, S. M., Worthey, G., \& González, J. J. 2000a, AJ, 119, 164

Trager, S. C., Faber, S. M., Worthey, G., \& González, J. J. 2000b, AJ, 120, 165

Véron-Cetty, M.-P., \& Véron, P. 2003, A\&A, 412, 399

Vorontsov-Velyaminov, B. A. 1959, Atlas and catalog of interacting galaxies 